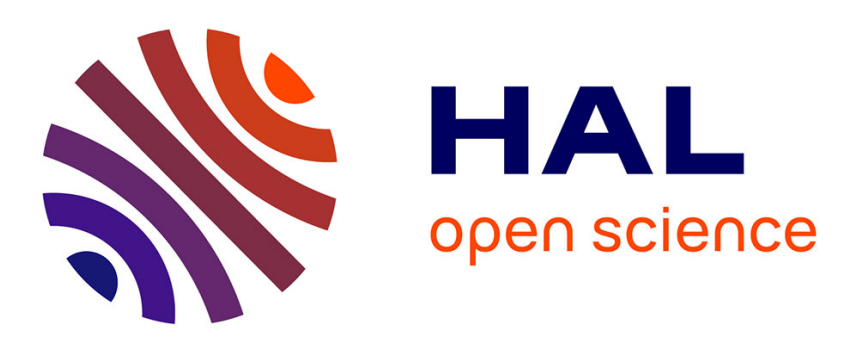

\title{
Computational and quasi-analytical models for non-linear vibrations of resonant MEMS and NEMS sensors
}

Najib Kacem, Sébastien Baguet, Sebastien Hentz, Régis Dufour

\section{- To cite this version:}

Najib Kacem, Sébastien Baguet, Sebastien Hentz, Régis Dufour. Computational and quasi-analytical models for non-linear vibrations of resonant MEMS and NEMS sensors. International Journal of Non-Linear Mechanics, 2011, 46 (3), pp.532-542. 10.1016/j.ijnonlinmec.2010.12.012 . hal-00633150

\author{
HAL Id: hal-00633150 \\ https://hal.science/hal-00633150
}

Submitted on 18 Sep 2014

HAL is a multi-disciplinary open access archive for the deposit and dissemination of scientific research documents, whether they are published or not. The documents may come from teaching and research institutions in France or abroad, or from public or private research centers.
L'archive ouverte pluridisciplinaire HAL, est destinée au dépôt et à la diffusion de documents scientifiques de niveau recherche, publiés ou non, émanant des établissements d'enseignement et de recherche français ou étrangers, des laboratoires publics ou privés. 
Computational and quasi-analytical models for nonlinear vibrations of resonant MEMS and NEMS sensors

N. Kacem, S. Baguet, S. Hentz, R. Dufour

PII: S0020-7462(10)00202-7

DOI: doi:10.1016/j.ijnonlinmec.2010.12.012

Reference: NLM 1800

www.elsevier.com/locate/nlm

To appear in: International Journal of NonLinear Mechanics

Received date: 11 May 2010

Revised date: 21 December 2010

Accepted date: 26 December 2010

Cite this article as: N. Kacem, S. Baguet, S. Hentz and R. Dufour, Computational and quasi-analytical models for nonlinear vibrations of resonant MEMS and NEMS sensors, International Journal of Non-Linear Mechanics, doi:10.1016/j.ijnonlinmec.2010.12.012

This is a PDF file of an unedited manuscript that has been accepted for publication. As a service to our customers we are providing this early version of the manuscript. The manuscript will undergo copyediting, typesetting, and review of the resulting galley proof before it is published in its final citable form. Please note that during the production process errors may be discovered which could affect the content, and all legal disclaimers that apply to the journal pertain. 


\title{
Computational and quasi-analytical models for nonlinear vibrations of resonant MEMS and NEMS sensors
}

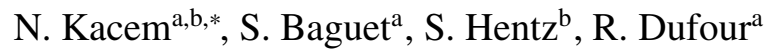 \\ ${ }^{a}$ Université de Lyon, CNRS \\ INSA-Lyon, LaMCoS UMR5259, F-69621, Villeurbanne, France \\ ${ }^{b}$ CEA/LETI - MINATEC, Grenoble, France
}

\begin{abstract}
Large-amplitude nonlinear vibrations of micro and nano electromechanical resonant sensors around their primary resonance are investigated. A comprehensive multiphysics model based on the Galerkin decomposition method coupled with the averaging method is developed in the case of electrostatically actuated clamped-clamped resonators. The model is purely analytical and includes the main sources of nonlinearities as well as fringing field effects. The influence of the higher modes and the validation of the model are demonstrated with respect to the shooting method as well as the harmonic balance coupled with the asymptotic numerical method. This model allows designers to investigate the sensitivity variation of resonant sensors in the nonlinear regime with respect to the electrostatic forcing.
\end{abstract}

Keywords:

MEMS, resonator, microbeam, nonlinear dynamics, reduced-order model, harmonic balance method, asymptotic numerical method

\section{Introduction}

Today, micro-electro-mechanical systems (MEMS) are in the heart of new technologies which are widely used in aerospace, automotive, biotechnology, instrumentation, robotics or manufacturing. Overcoming current technological challenges requires more and more reduced sizes. The next step in miniaturization is the so-called nano-electro-mechanical systems (NEMS). Operating

\footnotetext{
*Corresponding author. Tel.: +3343878 01 27; fax: +33438782434.

Email address: najib.kacem@insa-lyon.fr(N. Kacem)
} 
at the nanoscale, these systems come with many interesting physical attributes which makes them the best candidates for ultrasensitive mass and force detection [1-3]. The transition from MEMS to NEMS has implied many reconsiderations concerning the sensing techniques. Besides, in order to compensate the loss of performances when sensors are scaled down to the NEMS level, the resonant sensing has been widely implemented in nanosensors. This type of sensing basically consists in detecting a frequency shift due to an applied axial force to be measured while electrostatically exciting the resonator at its fundamental frequency.

The resolution of such a sensor is given by the minimum detectable frequency shift which is inversely proportional to the resonator drive amplitude. It is limited by the onset of nonlinearities which occur proportionally sooner with respect to the device size $[4,5]$. Moreover, below the bistability limit, it is extremely difficult to detect the oscillations of such small sensors. Hence, it is important to model the nonlinear dynamics of NEMS-based resonant sensors at large amplitudes.

The dynamic behavior of MEMS has been investigated by several authors so far. A survey of the electrostatic force modeling, a comprehensive review on MEMS modeling and an overview of the nonlinear dynamics of MEMS can be found in [6], [7] and [8]. Existing models are of variable complexity, depending on assumptions concerning the mechanical and electrostatic nonlinearities. For example a simple lumped spring-mass model was considered in $[9,10]$ and the continuous linear Euler-Bernoulli beam theory was used in [11]. Nevertheless, several studies pointed out the importance of deformation dependent mid-plane stretching for axially constrained microbeams undergoing large-amplitude vibration [12] and the nonlinear Euler-Bernoulli beam theory is therefore used in most recent studies [13-21]. The electrostatic force is usually modeled using the parallel-plate approximation together with correction terms accounting for the fringing field effects due to beam's finite width [22]. In order to facilitate the use of numerical or analytical methods, the nonlinearity of the electrostatic forcing term was further simplified by means of a high-order Taylor expansion in [10, 13, 21, 23] or a least squares approximation in [15].

Except for simplified models [9, 10, 20], analytical solutions are usually not obtainable for the continuous model. Some authors used finite-element simulations to study the vibrational behavior of MEMS either by means of a specific fully coupled finite element formulation [24] or a commercial software [20]. Reduced-order models are generally preferred due to their limited 
computational cost. The Galerkin method is used in [13, 15, 18, 19, 23], the simplified 1-mode Galerkin method in $[20,21]$ and the differential quadrature method (DQM) in [16, 17]. Several techniques have been proposed for solving the resulting nonlinear equations of motion, among which time integration [13, 19, 23, 24], perturbation methods [14], the shooting method [18], the invariant manifold method (or method of nonlinear normal forms) [15] or the homotopy analysis method which provides semi-analytic solutions [21].

In this paper, the nonlinear dynamics of micro and nano electromechanical resonant sensors are investigated at large amplitudes around their primary resonance. A comprehensive multiphysics model accounting for main sources of nonlinearities as well as fringing field effects and based on the Galerkin discretization is developed in the case of electrostatically actuated clamped-clamped resonators. An efficient numerical procedure for the continuation of periodic solutions based on the harmonic balance method (HBM) coupled with the asymptotic numerical method (ANM) is used to obtain the response curves of the resonator. A reduced-order purely analytical solution is then derived and validated with respect to the numerical HBM+ANM solution for several designs and working parameters. Finally, the interest of such an analytical solution is demonstrated though a study of the sensitivity of the resonator to axial force for acceleration sensing purpose.

\section{Model}

A clamped-clamped micro-beam as sketched in Fig. 1 is considered in the present study. It is actuated by a voltage $v(\tilde{t})=V_{d c}+V_{a c} \cos (\tilde{\Omega} \tilde{t})$, where $V_{d c}$ is the DC polarization voltage, $V_{a c}$ is the amplitude of the applied AC voltage, and $\tilde{\Omega}$ is the forcing frequency. A distributed electrostatic force is created between the beam and the rigid electrode, thus leading to the microbeam deformation and vibration.

\subsection{Equation of motion}

The equation of motion that governs the transverse deflection $\tilde{w}(\tilde{x}, \tilde{t})$ along $\mathrm{Z}$ axis is written as

$$
\rho b h \frac{\partial^{2} \tilde{w}}{\partial \tilde{t}^{2}}+\tilde{c} \frac{\partial \tilde{w}}{\partial \tilde{t}}+E I \frac{\partial^{4} \tilde{w}}{\partial \tilde{x}^{4}}-\tilde{N}(t) \frac{\partial^{2} \tilde{w}}{\partial \tilde{x}^{2}}=\tilde{F}_{e}
$$

where $\tilde{x}$ is the coordinate along the length $l$ of the micro-beam which is assumed sufficiently narrow, so that the cylindrical deformations can be neglected, $\tilde{t}$ is time, $E$ and $\rho$ are the effective 


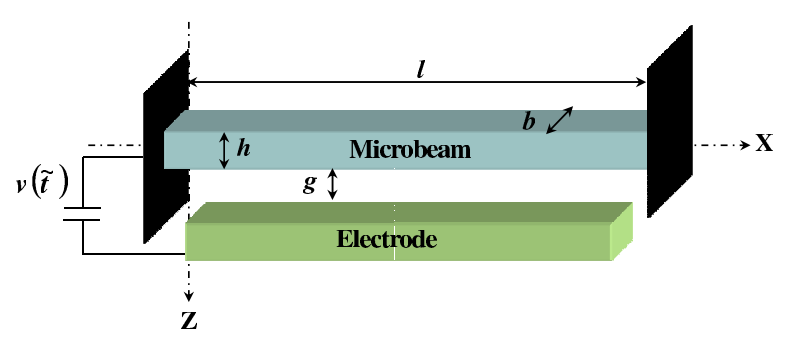

Figure 1: Schema of an electrically actuated micro-beam

Young's modulus and mass density of the beam material, $b$ and $h$ are the micro-beam thickness and width, $g$ is the capacitor gap width, i.e. the distance between the beam and the electrode at the undeformed state, $I=b h^{3} / 12$ is the moment of inertia of the rectangular cross-section, $\tilde{c}$ is the linear viscous damping per unit length, and $\varepsilon_{0}$ is the permittivity of the gap medium. In addition, $\tilde{N}(t)$ is the axial tension force

$$
\tilde{N}(t)=\tilde{N}_{0}+\frac{E b h}{2 l} \int_{0}^{l}\left[\frac{\partial \tilde{w}(\tilde{x}, \tilde{t})}{\partial \tilde{x}}\right]^{2} d \tilde{x}
$$

which consists of a constant applied tensile axial force $\tilde{N}_{0}$ and a nonlinear part due to stretching effects [25]. $\tilde{N}_{0}$ results from the residual stress on the silicon or the effect of the applied axial load which controls the natural frequency of the micro-beam. The right-hand side of Eq. (1) corresponds to the parallel-plate approximation of the electric force including the fringing field effects with respect to the beam width by means of the coefficient $C_{n}=1+1.9861\left(\frac{g}{b}\right)^{0.8258}$ [22] which has been validated using three-dimensional finite element simulations [4], where the assumption of neglected effect of the resonator thickness with respect to its transverse displacement has been verified.

$$
\tilde{F}_{e}(\tilde{x}, \tilde{t})=\frac{1}{2} \varepsilon_{0} \frac{b C_{n}\left[V_{d c}+V_{a c} \cos (\tilde{\Omega} \tilde{t})\right]^{2}}{(g-\tilde{w})^{2}}
$$

The beam is subject to the boundary conditions

$$
\tilde{w}(0, \tilde{t})=\tilde{w}(l, \tilde{t})=\frac{\partial \tilde{w}}{\partial \tilde{x}}(0, \tilde{t})=\frac{\partial \tilde{w}}{\partial \tilde{x}}(l, \tilde{t})=0
$$

\subsection{Normalization}

It is convenient to rescale equations by introducing the non-dimensional variables

$$
w=\frac{\tilde{w}}{g}, \quad x=\frac{\tilde{x}}{l}, \quad t=\frac{\tilde{t}}{\tau}
$$


where $\tau=\frac{2 l^{2}}{h} \sqrt{\frac{3 \rho}{E}}$. Substituting Eq. (5) into Eqs. (1) and (4), we obtain

$$
\begin{gathered}
\frac{\partial^{2} w}{\partial t^{2}}+c \frac{\partial w}{\partial t}+\frac{\partial^{4} w}{\partial x^{4}}-\left[N_{0}+\alpha_{1} \int_{0}^{1}\left(\frac{\partial w}{\partial x}\right)^{2} d x\right] \frac{\partial^{2} w}{\partial x^{2}}=\alpha_{2} \frac{\left[V_{d c}+V_{a c} \cos (\Omega t)\right]^{2}}{(1-w)^{2}} \\
w(0, t)=w(1, t)=\frac{\partial w}{\partial x}(0, t)=\frac{\partial w}{\partial x}(1, t)=0
\end{gathered}
$$

where

$$
c=\frac{\tilde{c} l^{4}}{E I \tau}, N_{0}=\frac{\tilde{N}_{0} l^{2}}{E I}, \alpha_{1}=6\left[\frac{g}{h}\right]^{2}, \alpha_{2}=\frac{6 \varepsilon_{0} C_{n} l^{4}}{E h^{3} g^{3}}, \Omega=\tilde{\Omega} \tau
$$

\subsection{Modal decomposition}

The beam deflection $w(x, t)$ can be written as a sum of a static DC displacement $w_{s}(x)$ and a time-varying AC displacement $w_{d}(x, t)$. However, for the considered devices, it is easy to check that the static deflection is negligible. Typically, the measured quality factors $Q$ are in the range $\left[10^{4}-5.10^{4}\right]$ and $V_{d c} \leq 200 V_{a c}$. Thus, the static/dynamic deflections ratio is

$$
\frac{w_{s}(x)}{w_{d}(x, t)} \approx \frac{V_{d c}}{2 Q \cdot V_{a c}} \leq 1 \%
$$

The Galerkin decomposition method based on linear undamped mode shapes is used to eliminate the spatial dependence and transform Eq. (6) into a multiple degree-of-freedom system (MDOF) consisting of ordinary differential equations in time. The deflection of the beam is approximated by the series

$$
w(x, t)=\sum_{k=1}^{N_{m}} a_{k}(t) \phi_{k}(x)
$$

where $a_{k}(t)$ is the $k^{\text {th }}$ time varying generalized coordinate and $\phi_{k}(x)$ is the $k^{\text {th }}$ linear undamped eigenmode solution of

$$
\frac{d^{4} \phi_{k}(x)}{d x^{4}}=\lambda_{k}^{4} \phi_{k}(x)
$$

Thus, the analytical form of the eigenmodes is given by

$$
\phi_{k}(x)=A_{k}\left\{\cos \lambda_{k} x-\cosh \lambda_{k} x+\left[\frac{\cosh \lambda_{k}-\cos \lambda_{k}}{\sin \lambda_{k}-\sinh \lambda_{k}}\right]\left[\sin \lambda_{k} x-\sinh \lambda_{k} x\right]\right\}
$$

with $\lambda_{k}$ solutions of the transcendental equation

$$
1-\cos \lambda_{k} \cosh \lambda_{k}=0
$$


These functions are a modal basis for the scalar product

$$
\langle u, v\rangle=\int_{0}^{1} u(x) v(x) d x
$$

and the coefficients $A_{k}$ are chosen to normalize the eigenmodes such that $\left\langle\phi_{i}, \phi_{j}\right\rangle=\delta_{i j}$.

\subsection{Galerkin procedure}

In many models in the literature, the electrostatic nonlinear forcing term is approximated by means of a Taylor development in order to simplify the Galerkin procedure $[13,15]$. However, when vibration amplitudes become large, such a development is no longer valid. Here, the complete contribution of the nonlinear electrostatic forces is included in the resonator dynamics without approximation since Eq. (6) is multiplied by $\phi_{i}(x)(1-w)^{2}$.

This method has some disadvantages like the non orthogonality of the operator $w^{4} \frac{\partial^{4} w}{\partial x^{4}}$ with respect to the undamped linear mode shapes of the resonator, the increase of the nonlinearity level in the normalized equation of motion (6) as well as the incorporation of new nonlinear terms such as the Van der Pol damping. Nevertheless, the resulting equation contains less parametric terms than if the nonlinear electrostatic forces were expanded in Taylor series and the solution of nonlinear problem is valid for large displacements of the beam up to the gap. The modal projection consists in substituting Eq. (10) in Eq. (6), using Eq. (11) to eliminate $d^{4} \phi_{k}(x) / d x^{4}$ and integrating 
the outcome from $x=0$ to 1 . Doing so, Eq. (6) becomes

$$
\begin{aligned}
& \ddot{a}_{i}-2 \sum_{j=1}^{N_{m}} \sum_{k=1}^{N_{m}}\left(\int_{0}^{1} \phi_{k} \phi_{j} \phi_{i} d x\right) a_{k} \ddot{a}_{j} \\
& +\sum_{j=1}^{N_{m}} \sum_{k=1}^{N_{m}} \sum_{l=1}^{N_{m}}\left(\int_{0}^{1} \phi_{l} \phi_{k} \phi_{j} \phi_{i} d x\right) a_{l} a_{k} \ddot{a}_{j} \\
& +c_{i} \dot{a}_{i}-2 \sum_{j=1}^{N_{m}} \sum_{k=1}^{N_{m}}\left(\int_{0}^{1} \phi_{k} \phi_{j} \phi_{i} d x\right) c_{j} a_{k} \dot{a}_{j} \\
& +\sum_{j=1}^{N_{m}} \sum_{k=1}^{N_{m}} \sum_{l=1}^{N_{m}}\left(\int_{0}^{1} \phi_{l} \phi_{k} \phi_{j} \phi_{i} d x\right) c_{j} a_{l} a_{k} \dot{a}_{j} \\
& +\lambda_{i}^{4} a_{i}-2 \sum_{j=1}^{N_{m}} \sum_{k=1}^{N_{m}}\left(\int_{0}^{1} \phi_{k} \phi_{j} \phi_{i} d x\right) \lambda_{j}^{4} a_{k} a_{j} \\
& +\sum_{j=1}^{N_{m}} \sum_{k=1}^{N_{m}} \sum_{l=1}^{N_{m}}\left(\int_{0}^{1} \phi_{l} \phi_{k} \phi_{j} \phi_{i} d x\right) \lambda_{j}^{4} a_{l} a_{k} a_{j} \\
& -\left[N_{0}+\alpha_{1}\left(\sum_{m=1}^{N_{m}} \sum_{n=1}^{N_{m}}\left(\int_{0}^{1} \phi_{n}^{\prime} \phi_{m}^{\prime} d x\right) a_{n} a_{m}\right)\right] \\
& {\left[\sum_{j=1}^{N_{m}}\left(\int_{0}^{1} \phi_{j}^{\prime \prime} \phi_{i} d x\right) a_{j}\right.} \\
& -2 \sum_{j=1}^{N_{m}} \sum_{k=1}^{N_{m}}\left(\int_{0}^{1} \phi_{k} \phi_{j}^{\prime \prime} \phi_{i} d x\right) a_{k} a_{j} \\
& \left.+\sum_{j=1}^{N_{m}} \sum_{k=1}^{N_{m}} \sum_{l=1}^{N_{m}}\left(\int_{0}^{1} \phi_{l} \phi_{k} \phi_{j}^{\prime \prime} \phi_{i} d x\right) a_{l} a_{k} a_{j}\right] \\
& =\alpha_{2}\left(V_{d c}+V_{a c} \cos \Omega t\right)^{2}\left(\int_{0}^{1} \phi_{i} d x\right) \\
& \text { for } i=1,2, \ldots, N_{m}
\end{aligned}
$$


Eq. (15) can be written in matrix-vector form as

$$
\begin{aligned}
& {\left[\mathbf{M}_{0}+\mathbf{M}_{1}(\mathbf{a})+\mathbf{M}_{2}(\mathbf{a})\right] \ddot{\mathbf{a}}} \\
& +\left[\mathbf{C}_{0}+\mathbf{C}_{1}(\mathbf{a})+\mathbf{C}_{2}(\mathbf{a})\right] \dot{\mathbf{a}} \\
& +\left[\mathbf{K}_{0}+\mathbf{K}_{1}(\mathbf{a})+\mathbf{K}_{2}(\mathbf{a})\right] \mathbf{a} \\
& -\left[N_{0}+\alpha_{1} T_{2}(\mathbf{a})\right]\left[\mathbf{K}_{T}+\mathbf{K}_{T 1}(\mathbf{a})+\mathbf{K}_{T 2}(\mathbf{a})\right] \mathbf{a} \\
& =\alpha_{2}\left(V_{d c}+V_{a c} \cos \Omega t\right)^{2} \mathbf{F}
\end{aligned}
$$

with $\mathbf{a}(t)=\left[a_{1}(t), a_{2}(t), \ldots, a_{N_{m}}(t)\right]^{T}$. The components of matrices $\mathbf{M}_{0}, \mathbf{M}_{1}, \mathbf{M}_{2}, \mathbf{C}_{0}, \mathbf{C}_{1}, \mathbf{C}_{2}, \mathbf{K}_{0}$, $\mathbf{K}_{1}, \mathbf{K}_{2}, \mathbf{K}_{T}, \mathbf{K}_{T 1}$ and $\mathbf{K}_{T 2}$ are respectively $M_{i j}, M_{1 i j}, M_{2 i j}, C_{i j}, C_{1 i j}, C_{2 i j}, K_{i j}, K_{1 i j}, K_{2 i j}, K_{T i j}, K_{T 1 i j}$, $K_{T 2 i j}$ with

$$
\begin{aligned}
& M_{0 i j}=\delta_{i j} \\
& M_{1 i j}=-2 \sum_{k=1}^{N_{m}}\left(\int_{0}^{1} \phi_{k} \phi_{j} \phi_{i} d x\right) a_{k} \\
& M_{2 i j}=\sum_{k=1}^{N_{m}} \sum_{l=1}^{N_{m}}\left(\int_{0}^{1} \phi_{l} \phi_{k} \phi_{j} \phi_{i} d x\right) a_{l} a_{k} \\
& C_{0 i j}=c_{i} \delta_{i j} \quad C_{1 i j}=c_{j} M_{1 i j} \quad C_{2 i j}=c_{j} M_{2 i j} \\
& K_{0 i j}=\lambda_{i}^{4} \delta_{i j} \quad K_{1 i j}=\lambda_{j}^{4} M_{1 i j} \quad K_{2 i j}=\lambda_{j}^{4} M_{2 i j} \\
& K_{T i j}=\int_{0}^{1} \phi_{j}^{\prime \prime} \phi_{i} d x \\
& K_{T 1 i j}=-2 \sum_{k=1}^{N_{m}}\left(\int_{0}^{1} \phi_{k} \phi_{j}^{\prime \prime} \phi_{i} d x\right) a_{k} \\
& K_{T 2 i j}=\sum_{k=1}^{N_{m}} \sum_{l=1}^{N_{m}}\left(\int_{0}^{1} \phi_{l} \phi_{k} \phi_{j}^{\prime \prime} \phi_{i} d x\right) a_{l} a_{k}
\end{aligned}
$$

The scalar $T_{2}(\mathbf{a})$ and the entries of vector $\mathbf{F}$ are respectively

$$
\begin{aligned}
& T_{2}(\mathbf{a})=\sum_{m=1}^{N_{m}} \sum_{n=1}^{N_{m}}\left(\int_{0}^{1} \phi_{n}^{\prime} \phi_{m}^{\prime} d x\right) a_{n} a_{m} \\
& F_{i}=\int_{0}^{1} \phi_{i} d x
\end{aligned}
$$

Indices 1 and 2 for matrices $\mathbf{M}, \mathbf{C}, \mathbf{K}, \mathbf{K}_{T}$ and for $T_{2}$ denote nonlinearities of order 1 and 2 with 
respect to a. Once all these coefficients have been computed, system (16) can be solved with a suitable numerical procedure.

\section{High order Harmonic Balance Method combined with the Asymptotic Numerical Method (HBM+ANM)}

The Harmonic Balance Method (HBM) is commonly used for computing periodic solutions. It consists in assuming a time solution in the form of a Fourier series and comparing/balancing the coefficients of the same harmonic components. In this way, non linear differential equations in the space variables and time are transformed into a nonlinear algebraic system in the space variables and frequency. However, when nonlinearities are complicated, the derivation of the algebraic system becomes very cumbersome. Alternative methods have been proposed to overcome these shortcomings, such as the incremental harmonic balance method (IHBM) [26] or the alternating frequency/time domain harmonic balance method (AFT) [27] but they are very demanding from a computational point of view.

Recently, Cochelin et al. [28] have proposed another strategy for applying the classical HBM with a large number of harmonics. The basic idea consists in recasting the original system (16) into a new system where nonlinearities are at most quadratic polynomials by introducing as many new variables as needed. This leads to an augmented, but quadratic only, nonlinear system for which the application of the HBM is quite straightforward. Furthermore, this quadratic framework makes it possible to use the so-called Asymptotic Numerical Method (ANM) for the continuation of solutions. The ANM consists in computing power series expansions of solution branches and presents several advantages: it provides continuous solutions, the continuation is very robust, and the control of the step length is automatic and always optimal [29-32].

This method is detailled in [28]. Its application to our nonlinear differential system (16) is detailled hereafter. The association of the HBM and the ANM will be subsequently referred to as $\mathrm{HBM}+\mathrm{ANM}$. 


\subsection{Quadratic recast}

The key point of this method lies in the quadratic recast of Eq. (16) by introducing the following set of auxiliary variables,

$$
\begin{array}{rlrl}
\mathbf{y} & =\dot{\mathbf{a}} & & \left(\operatorname{size} N_{m}\right) \\
\mathbf{z} & =\ddot{\mathbf{a}}=\dot{\mathbf{y}} & \left(\operatorname{size} N_{m}\right) \\
\mathbf{M}_{t o t} & =\mathbf{M}_{1}(\mathbf{a})+\mathbf{M}_{2}(\mathbf{a}) & \left(\operatorname{size} N_{m}^{2}\right) \\
\mathbf{K}_{T t o t} & =\mathbf{K}_{T 1}(\mathbf{a})+\mathbf{K}_{T 2}(\mathbf{a}) & \left(\operatorname{size} N_{m}^{2}\right) \\
\mathbf{S} & =\mathbf{K}_{T t o t} \mathbf{a} & & \left(\operatorname{size} N_{m}\right) \\
T & =T_{2}(\mathbf{a}) & & (\operatorname{size} 1)
\end{array}
$$

system (16) can be rewritten as

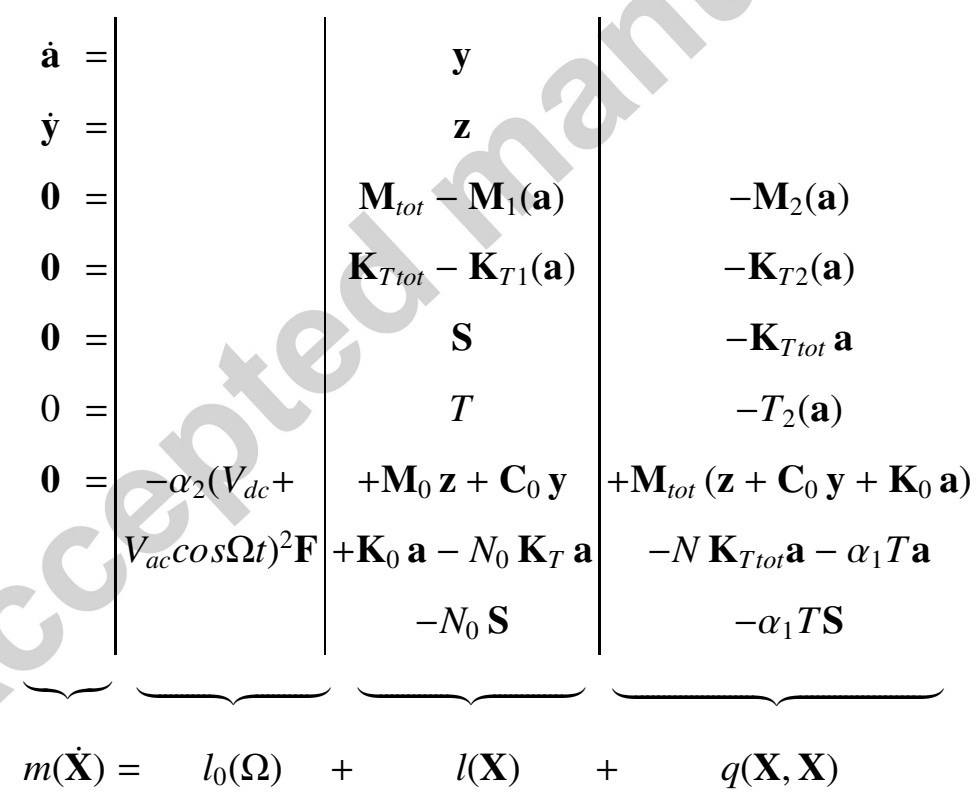

where $\mathbf{X}=\left(\mathbf{a}, \mathbf{y}, \mathbf{z}, \mathbf{M}_{\text {tot }}, \mathbf{K}_{T t o t}, \mathbf{S}, T\right)^{T}$ is the unknown vector of size $N_{e q}=2 N_{m}^{2}+4 N_{m}+1$, in which matrices $\mathbf{M}_{t o t}$ and $\mathbf{K}_{T t o t}$ are reshaped as vectors. $l_{0}$ is a constant vector with respect to $\mathbf{X}, l(\cdot)$ and $m(\cdot)$ are linear vector valued operators with respect to $\mathbf{X}$, and $q(\cdot, \cdot)$ is a quadratic vector valued operator. 


\subsection{Application of the HBM}

The HBM consists in decomposing $\mathbf{X}(t)$ into a truncated Fourier series

$$
\mathbf{X}(t)=\mathbf{X}_{0}+\sum_{k=1}^{H} \mathbf{X}_{k}^{c} \cos (k \Omega t)+\sum_{k=1}^{H} \mathbf{X}_{k}^{s} \sin (k \Omega t)
$$

and inserting this expansion in the nonlinear differential system (20). By balancing the first $2 \mathrm{H}+$ 1 harmonic terms and neglecting the higher order harmonics, the following nonlinear algebraic system of size $(2 H+1) \times N_{e q}$ is obtained

$$
\Omega M(\mathbf{U})=L_{0}+L(\mathbf{U})+Q(\mathbf{U}, \mathbf{U})
$$

where $\mathbf{U}=\left[\mathbf{X}_{0}, \mathbf{X}_{1}^{c T}, \mathbf{X}_{1}^{s T}, \ldots, \mathbf{X}_{H}^{c T}, \mathbf{X}_{H}^{s T}\right]^{T}$ contains the components of the Fourier series (21). The new operators $L_{0}, M(\cdot), L(\cdot)$ and $Q(\cdot, \cdot)$ depend only on the operators $l_{0}, m(\cdot), l(\cdot)$ and $q(\cdot, \cdot)$. Their explicit expressions can be found in Cochelin et al. [28].

\subsection{Continuation by the ANM}

In order to apply the Asymptotic Numerical Method, Eq. (22) is reformulated as

$$
\mathbf{R}(\mathbf{U}, \Omega)=L_{0}+L(\mathbf{U})+Q(\mathbf{U}, \mathbf{U})-\Omega M(\mathbf{U})=0
$$

Assuming that a regular solution $\left(\mathbf{U}_{0}, \Omega_{0}\right)$ of Eq. (22) is known, the branch of solution starting at $\left(\mathbf{U}_{0}, \Omega_{0}\right)$ is represented as a power series expansion (truncated at order $N$ ) with respect to the path-parameter $\eta[32]$

$$
\begin{aligned}
& \mathbf{U}(\eta)=\mathbf{U}_{0}+\eta \mathbf{U}_{1}+\eta^{2} \mathbf{U}_{2}+\ldots+\eta^{N} \mathbf{U}_{N} \\
& \Omega(\eta)=\Omega_{0}+\eta \Omega_{1}+\eta^{2} \Omega_{2}+\ldots+\eta^{N} \Omega_{N}
\end{aligned}
$$

If the pseudo-arclength definition is chosen, the path-parameter $a$ can be expressed as [30]

$$
\eta=\mathbf{U}_{1} \cdot\left(\mathbf{U}-\mathbf{U}_{0}\right)^{T}+\Omega_{1}\left(\Omega-\Omega_{0}\right)
$$

where $\left(\mathbf{U}_{1}, \Omega_{1}\right)$ is the tangent vector at $\left(\mathbf{U}_{0}, \Omega_{0}\right)$. Introducing the series expansions (24) into Eqs. (23) and (25) and keeping the power terms up to order $N$ leads to

$$
\mathbf{R}(\eta)=\mathbf{R}_{0}+\eta \mathbf{R}_{1}+\eta^{2} \mathbf{R}_{2}+\ldots+\eta^{N} \mathbf{R}_{N}=0
$$


Equating the terms $\mathbf{R}_{i(1 \leq i \leq N)}$ to zero permits transforming the nonlinear system (23) into $N$ successive linear systems of $N_{e q}$ equations, that are then solved recursively in order to obtain $\mathbf{U}_{i}$ and $\Omega_{i}$ $(1 \leq i \leq N)$. This is very efficient from a computational point of view since all the linear systems share the same matrix which corresponds to the Jacobian of $\mathbf{R}$ evaluated at $\left(\mathbf{U}_{0}, \Omega_{0}\right)$. Only the right-hand side vector changes with the order. Moreover, the range of validity $a_{\max }$ of the series can be approximated a priori by

$$
\eta_{\text {max }}=\left(\frac{\varepsilon}{\mathbf{R}_{N+1}}\right)^{\frac{1}{N+1}}
$$

where $\varepsilon$ is a user-defined tolerance parameter. Thus a part of the solution branch is obtained by following Eq. (24) until $\eta=\eta_{\max }$. This end point is then used as a new starting point $\left(\mathbf{U}_{0}, \Omega_{0}\right)$ and the next part of the solution branch is obtained by restarting the continuation process. As a consequence, the step length is naturally adaptive and optimal [31] and the continuation is very robust.

\subsection{Stability analysis}

A stability analysis can be conducted during the continuation by studying the complex eigenvalues (Floquet multipliers) of the Monodromy matrix $\mathcal{M}$ associated with the periodic solution of system (20). This Monodromy matrix is obtained by time integration over one period of the differential system

$$
\dot{\mathcal{M}}=\mathbf{J} \mathcal{M}
$$

where $\mathbf{J}$ is the Jacobian matrix of (20) and with the identity matrix as initial condition. The solution is stable if all the multipliers lie inside the complex unit circle. It is unstable otherwise [33, 34].

\section{Simplified analytical model}

\subsection{Mode properties and approximate integrals}

Using the change of variable $z=x-\frac{1}{2}$ for all the eigenmodes, it is easy to check that $\Delta: z=0$ represents an axis of symmetry for all the odd modes and an axis of antisymmetry for all the even modes. Thus, the odd modes are even functions and the even modes are odd functions $\forall z \in$ 
$\left[-\frac{1}{2}, \frac{1}{2}\right]$. Consequently:

$$
\begin{array}{r}
\forall k \in \mathbb{N}^{*}, \forall i \in \mathbb{N}^{*} \text { and } \forall z \in\left[-\frac{1}{2}, \frac{1}{2}\right] \\
\int_{0}^{1} \Phi_{2 i} d x=\int_{0}^{1} \Phi_{2 i} \Phi_{k}^{2} d x=\int_{0}^{1} \Phi_{2 i} \Phi_{2 k-1}^{3} d x=0 \\
\int_{0}^{1} \Phi_{2 i-1} \Phi_{2 k}^{3} d x=\int_{0}^{1} \Phi_{2 i} \Phi_{k} \Phi_{k}^{\prime \prime} d x=0 \\
\int_{0}^{1} \Phi_{2 i}^{\prime} \Phi_{2 k-1}^{\prime} d x=\int_{0}^{1} \Phi_{2 i-1}^{\prime} \Phi_{2 k}^{\prime} d x=0 \\
\int_{0}^{1} \Phi_{2 i} \Phi_{2 k-1}^{2} \Phi_{2 k-1}^{\prime \prime} d x=\int_{0}^{1} \Phi_{2 i-1} \Phi_{2 k}^{2} \Phi_{2 k}^{\prime \prime} d x=0
\end{array}
$$

As a first approximation, the eigenvalues $\lambda_{k}$ which are solutions of the transcendental Eq. (13), can be written as:

$$
\forall k \geq 1 \quad \lambda_{k} \simeq \frac{[2 k+1] \pi}{2}
$$

Consequently:

$$
\forall k \geq 1 \quad \begin{aligned}
& \cos \left[\lambda_{k}\right] \simeq \sin \left[2 \lambda_{k}\right] \simeq 0 \\
& \sin \left[\lambda_{k}\right] \simeq[-1]^{k} \\
& \cos \left[2 \lambda_{k}\right] \simeq-1
\end{aligned}
$$

Moreover, $\cosh \left[\lambda_{k}\right] \sim \sinh \left[\lambda_{k}\right]$ in $\left[\lambda_{1},+\infty\right]$. One can check that the validity of this equivalence starts from the first mode. In fact, for $k=1, \lambda_{k} \simeq 4.73$ and $\cosh \left[\lambda_{1}\right] \simeq \sinh \left[\lambda_{1}\right] \simeq 56.65$.

In order to simplify the different integrals of Eqs. (17)-(18), Eqs. (30) and (31) are used combined with the equivalence $\cosh \left[\lambda_{k}\right] \sim \sinh \left[\lambda_{k}\right]$. Then, these integrals are approximated as equivalent to their limit when the unbounded function $\cosh \left[\lambda_{k}\right] \rightarrow+\infty$. Thus, approximate analytical closedform expressions of the different integrals are obtained with respect to the mode number (see Appendix). 


\subsection{Reduced order model}

Assuming that the first mode should be the dominant mode of the system, only one mode is retained $\left(N_{m}=1\right)$ and the other modes are neglected. Equation (16) becomes :

$$
\begin{aligned}
& \ddot{a}_{1}+\left(500.564+12.3 N_{0}\right) a_{1}+\left(927+28 N_{0}+151 \alpha_{1}\right) a_{1}^{3} \\
& +347 \alpha_{1} a_{1}^{5}+\left(1330.9+38.3 N_{0}\right) a_{1}^{2}+471 \alpha_{1} a_{1}^{4} \\
& +2.66 c_{1} a_{1} \dot{a}_{1}+1.85 c_{1} a_{1}^{2} \dot{a}_{1}+c_{1} \dot{a}_{1}+2.66 a_{1} \ddot{a}_{1} \\
& +1.85 a_{1}^{2} \ddot{a}_{1}=-\frac{8}{3 \pi} \alpha_{2}\left[V_{d c}+V_{a c} \cos (\Omega t)\right]^{2}
\end{aligned}
$$

To analyse the equation of motion (32), it proves convenient to invoke perturbation techniques which work well with the assumptions of "small" excitation and damping, typically valid in MEMS resonators. To facilitate the perturbation approach, in this case the method of averaging [33], a standard constrained coordinate transformation is introduced by assuming a slowly time-varying amplitude:

$$
\left\{\begin{array}{l}
a_{1}=A(t) \cos [\Omega t+\beta(t)] \\
\dot{a}_{1}=-A(t) \Omega \sin [\Omega t+\beta(t)] \\
\ddot{a}_{1}=-A(t) \Omega^{2} \cos [\Omega t+\beta(t)]
\end{array}\right.
$$

In addition, since near-resonant behavior is the principal operating regime of the proposed system, a detuning parameter $\sigma$ is introduced, as given by:

$$
\Omega=\omega_{n}+\varepsilon \sigma
$$

Separating the resulting equations and averaging them over the period $\frac{2 \pi}{\Omega}$ in the $t$-domain results in the system's averaged equations, in terms of amplitude and phase, which are given by:

$$
\begin{array}{r}
\dot{A}=-\frac{1}{2} \varepsilon \xi_{0} A-\frac{1}{8} \varepsilon \xi_{2} A^{3}-\frac{1}{2} \varepsilon \frac{\kappa}{\omega_{n}} \sin \beta+O\left(\varepsilon^{2}\right) \\
A \dot{\beta}=A \sigma \varepsilon-\frac{3}{8} \varepsilon \frac{\chi_{3}}{\omega_{n}} A^{3}-\frac{5}{16} \varepsilon \frac{\chi_{5}}{\omega_{n}} A^{5}+\frac{7}{10} \varepsilon \omega_{n} A^{3} \\
+\frac{1}{2} \varepsilon \frac{\kappa}{\omega_{n}} \cos \beta+O\left(\varepsilon^{2}\right)
\end{array}
$$

where

$$
\omega_{n}=\sqrt{500.564+12.3 N_{0}}
$$


and the other parameters are $\xi_{0}=c_{1}, \xi_{2}=1.85 c_{1}, \chi_{3}=927+28 N+151 \alpha_{1}, \chi_{5}=347 \alpha_{1}$ and $\kappa=\frac{16}{3 \pi} \alpha_{2} V_{a c} V_{d c}$.

The steady-state motions occur when $\dot{A}=\dot{\beta}=0$, which corresponds to the singular points of Eqs. (35) and (36). Thus, the frequency-response equation can be written in its implicit form as:

$$
\left(\frac{3 \chi_{3}}{4 \omega_{n}} A^{2}+\frac{5 \chi_{5}}{8 \omega_{n}} A^{4}-\frac{7 \omega_{n}}{5} A^{2}-2 \sigma\right)^{2}+\left(\xi_{0}+\frac{\xi_{2}}{4} A^{2}\right)^{2}=\left(\frac{\kappa}{A \omega_{n}}\right)^{2}
$$

The normalized displacement $W_{\max }$ with respect to the gap at the middle of the beam and the forcing frequency $\Omega$ can be expressed in function of the phase $\beta$. Thus, the frequency response curve can be plotted parametrically.

This analytical model is a lightweight, easy-to-use and very efficient tool for MEMS designers. Although it is very simple, it is able to capture all the nonlinear phenomena in the resonator dynamics (hardening and softening behaviors) and describe the competition between them.

\section{Results and discussion}

For all the numerical simulations with the ANM+HBM, only symmetric modes were considered due to the symmetry of the problem. It was verified also numerically that antisymmetric modes do not participate in the global dynamics of the resonator. In other words, a computation with 3 modes uses the odd modes 1,3 and 5. Two resonator designs were used, which have different size and quality factor $Q$ as listed in Table 1 . The ratio between the $A C$ and $D C$ voltages was set at $V_{a c}=0.1 V_{d c}$ for all simulations.

\begin{tabular}{cccccc}
\hline Resonator & $L(\mu m)$ & $b(\mu m)$ & $h(\mu m)$ & $g(\mu m)$ & $Q$ \\
\hline Design 1 & 400 & 10 & 10 & 2 & 10000 \\
Design 2 & 50 & 1 & 1 & 0.4 & 1000 \\
\hline
\end{tabular}

Table 1: Design parameters of investigated resonators.

\subsection{Validation of the numerical solution}

The dynamic response of the resonator obtained by HBM+ANM continuation is plotted in Fig. 2 for design 1 and an actuation voltage $V_{d c}=9 \mathrm{~V}$, thus $V_{a c}=0.9 \mathrm{~V}$. $W_{\max }$ is the displacement 


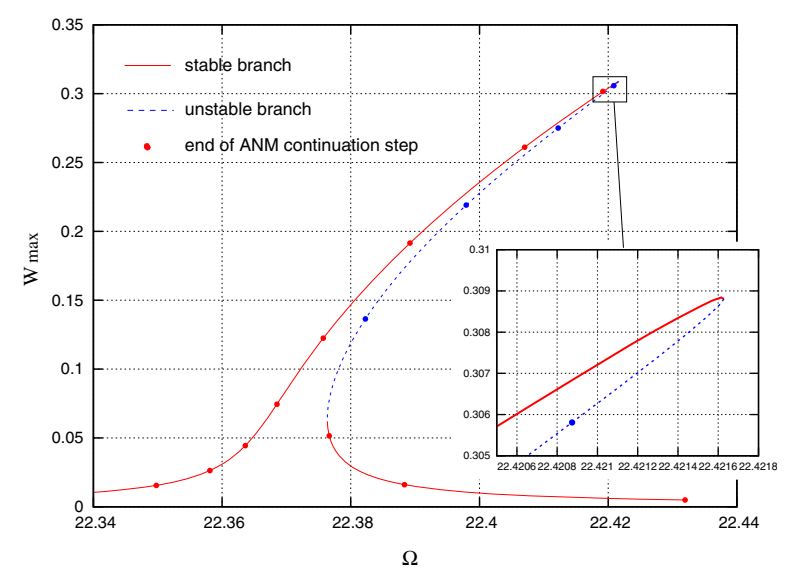

Figure 2: HBM+ANM continuation on a strongly nonlinear behavior (Design $1, V_{d c}=9 \mathrm{~V}, V_{a c}=0.9 \mathrm{~V}$ ), $N_{m}=1, H=7$, $N=30$.

of the beam normalized by the gap $g$ at its middle point $x=l / 2$. For this set of parameters, the resonator exhibits a strongly hardening behavior. The solution was computed with $N_{m}=1$ mode for the Galerkin procedure, $H=7$ harmonics for the $\mathrm{HBM}$ and the series truncated at order $N=30$ for the ANM. It can be observed that the high order polynomial expansions of the ANM continuation permit the description of the complete resonance curve with only 15 continuation steps and going through the sharp turning point with no difficulty. Unlike the classical continuation methods based on the Newton-Raphson prediction-correction procedure, the solution curve can be accurately evaluated and plotted between two consecutive ends of step. Moreover, the stability analysis can also be conducted for any value of the continuation parameter $\eta$ during one step and therefore the transition between stable and unstable branches can be precisely observed even if it occurs between two consecutive ends of step.

In order to validate the HBM+ANM, the shooting method was also implemented to be used as a reference solution. For the sake of conciseness, the implementation of the well-known shooting method is not detailed herein but can be found in [33] for instance. The confrontation is shown in Figs. 3 and 4 on design 1 described in Table 1 for electrostatic forcing leading to slightly $\left(V_{d c}=5 \mathrm{~V}\right.$, $\left.V_{a c}=0.5 \mathrm{~V}\right)$ and strongly $\left(V_{d c}=9 \mathrm{~V}, V_{a c}=0.9 \mathrm{~V}\right)$ nonlinear behaviors respectively. For each case, the number of modes $N_{m}$ retained in the simulation and the number of harmonics $H$ used for the HBM are varied in order to study both the influence of $N_{m}$ and the convergence of the HBM+ANM 

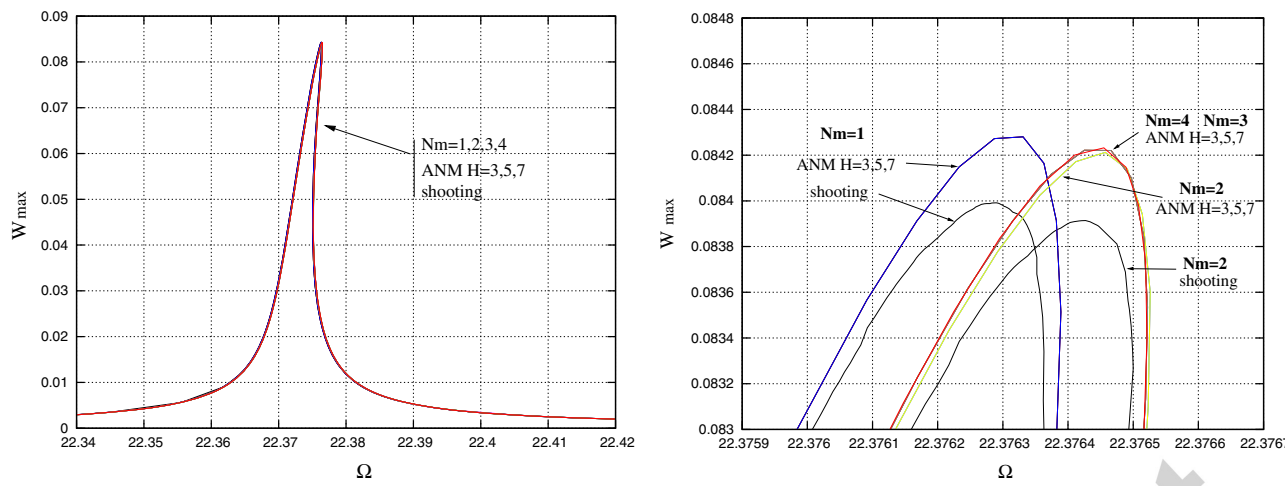

Figure 3: Confrontation Shooting $/ \mathrm{HBM}+\mathrm{ANM}$ on a slightly nonlinear behavior (Design $\left.1, V_{d c}=5 \mathrm{~V}, V_{a c}=0.5 \mathrm{~V}\right)$.
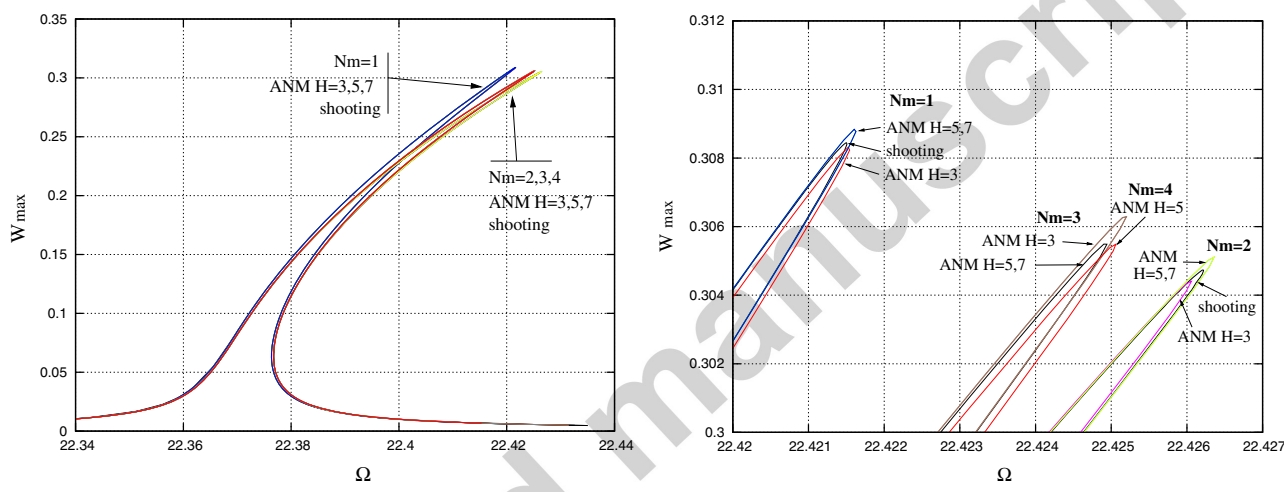

Figure 4: Second confrontation Shooting/HBM+ANM on a strongly nonlinear behavior (Design $1, V_{d c}=9 \mathrm{~V}$, $\left.V_{a c}=0.9 \mathrm{~V}\right)$.

according to $H$. In the slightly nonlinear case of Fig. 3, the convergence of the HBM+ANM is achieved with only 3 harmonics and results are in very good agreement with the shooting method. The slight difference between the curves obtained by shooting and HBM+ANM is due to the numerical precision used for the time-integration scheme of the shooting procedure. Concerning the number of modes, the results are identical with 2, 3 or 4 modes and the difference between 1 and 2 modes is negligible when compared to the peak frequency. In the strongly nonlinear case of Fig. 4, a small difference between the curves for 1 mode and 2, 3 or 4 modes is noticeable. Nevertheless, it is less than $0.1 \%$ with respect to the peak frequency, which is negligible compared to the frequency shifts induced by the fabrication tolerances. Again, the results are in good agreement between HBM+ANM and shooting methods, and 3 harmonics are sufficient to achieve 
a very good precision. It is important to underline that despite the higher number of equations to be solved (255 equations for the HBM+ANM instead of 2 equations for the shooting in the case $N_{m}=2 \mathrm{H=7}$ ), the HBM+ANM is much faster than shooting and its continuation also proves to be more robust as it does not require the development of specific adaptive step length strategies.

\subsection{Validation of the analytical model}

As previously demonstrated with the HBM+ANM, the errors made by using only the first mode in the Galerkin method are negligible with respect to errors due to others constraints such as fabrication, temperature, pressure... Thus one can expect that the reduced order analytical model should give satisfying results. For this purpose, the validation is ensured through comparisons with the HBM+ANM results.

Figure 5 shows a first confrontation between both methods on design 1 of Table 1 at a slight nonlinear regime corresponding to a $D C$ voltage $V_{d c}=5 \mathrm{~V}$. Analytical and numerical results are in good agreement. Particularly, at this nonlinear level, there is no notable difference between one, two or three modes for the HBM+ANM model. Indeed, the slight variations reported here between the resonance curves are below 15 parts per million ( $\mathrm{ppm}$ ) in resonance frequency and less than $1 \%$ in peak amplitude, which are very low compared to $0.5 \%$ in frequency due to residual stress [35] or micromachining imperfections as well as 5\% in peak amplitude due to the variation of the quality factor by ohmic losses [36] for a reasonable $D C$ voltage range. Obviously, the coupling between the different modes is extremely low in this case and the influence of higher modes is negligible.

Then, the polarization voltage is increased up to $9 \mathrm{~V}$. Consequently, the dynamic behavior of the resonator becomes strongly nonlinear as shown in Fig. 6 for peak amplitudes between 20\% and $35 \%$ of the gap. In these configurations, the coupling between the modes is strongly amplified. Nevertheless, the error between the analytical model and the HBM+ANM model is still negligible, even with respect to the computational solution with 3 modes (frequency shift $<0.1 \%$ ).

Finally, for confirmation, the same investigations have been made on a smaller resonator with a different geometry described in Table 1 (Design 2). Figure 7 displays the confrontation of both models at a high nonlinear regime for a polarization voltage $V_{d c}=5 \mathrm{~V}\left(V_{a c}=0.5 \mathrm{~V}\right)$. The same 


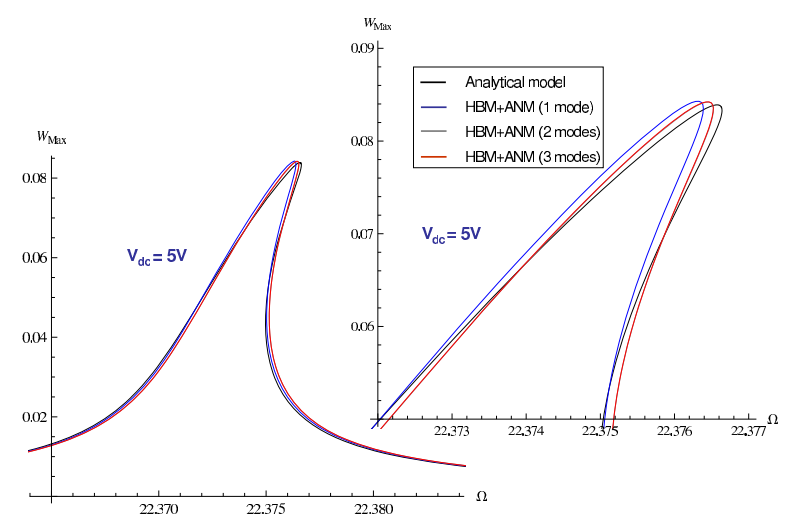

Figure 5: Confrontation HBM+ANM/Analytical model on a slightly nonlinear behavior (Design $1, V_{d c}=5 \mathrm{~V}$, $\left.V_{a c}=0.5 \mathrm{~V}\right)$.

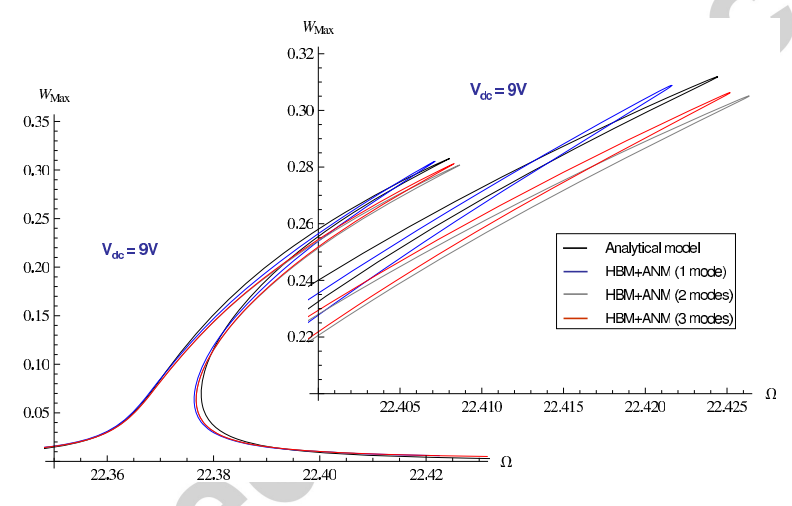

Figure 6: Confrontation $\mathrm{HBM}+\mathrm{ANM} /$ Analytical model on a strongly nonlinear behavior $\left(\right.$ Design $1, V_{d c}=9 \mathrm{~V}$, $\left.V_{a c}=0.9 \mathrm{~V}\right)$.

conclusions as before can be drawn concerning accuracy, which completes the analytical model validation.

The analytical model has the advantage to be fast and accurate. Furthermore, with this model it is possible to perform analytical parametric investigations with respect to the phase of the resonator oscillation and thus to derive analytical expressions that can be used as design rules by MEMS and NEMS designers in order to enhance the performances of resonant sensors.

\subsection{Sensitivity to axial force - Acceleration sensing}

Resonators can be used for acceleration sensing. Basically, as shown in Fig. 8, a resonant accelerometer consists in a proof mass $M$, suspended on the substrate by anchors (made of narrow 


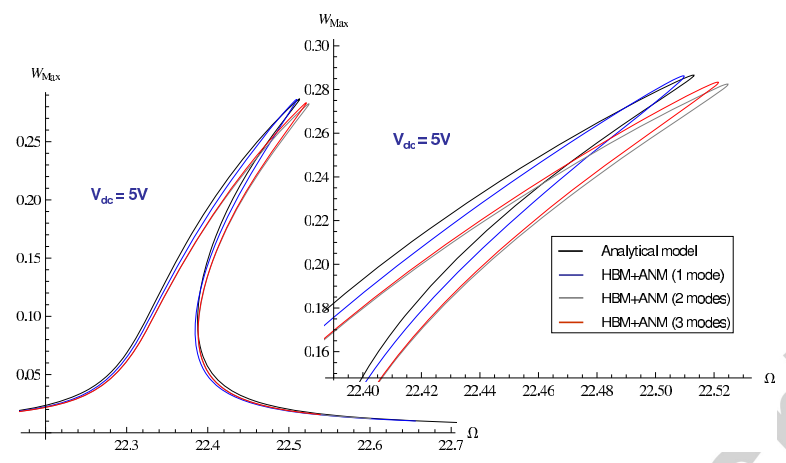

Figure 7: Confrontation HBM+ANM/Analytical model on a strongly nonlinear behavior (Design $2, V_{d c}=5 \mathrm{~V}$, $\left.V_{a c}=0.5 \mathrm{~V}\right)$.

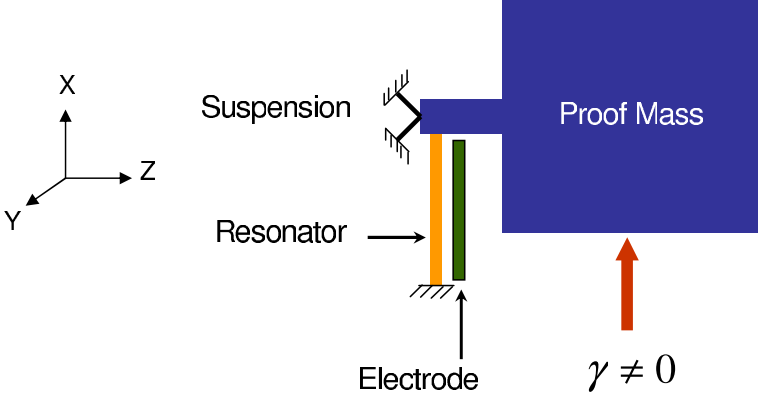

Figure 8: Resonant accelerometer structure 
beams), and a resonator placed close to the intersection of the anchors. The considered resonator is a clamped-clamped micro/nanobeam electrostatically actuated. An external acceleration $\gamma$ that is applied to the proof mass along the sensitive $X$-axis of the device, results in a force communicated axially onto the resonator. The applied axial force induces a shift in the resonator frequency due to a change in the nominal stored potential energy of the system. Thus, by evaluating the frequency shift, the acceleration applied to the device can be estimated.

The sensitivity or scale factor of the device is the constant of proportionality relating the input (acceleration) and output (frequency shift). Since the relation between the acceleration $\gamma$ and the communicated force to the resonator is linear, the scale factor in term of axial force can be used for parametric investigations with respect to the drive voltage when the resonator is driven in the nonlinear regime. By using the analytical model in its parametric form with respect to the phase $\beta$, the relative sensitivity of the resonator to an axial force can be written as:

$$
S F=\frac{\Delta f / f}{\Delta \tilde{N}_{0}}=\frac{1}{f\left(\beta=\frac{\pi}{2}, \tilde{N}_{0}=0\right)} \frac{d f\left(\beta=\frac{\pi}{2}, \tilde{N}_{0}\right)}{d \tilde{N}_{0}}
$$

with the forcing frequency $f=\Omega / 2 \pi, \Omega$ computed from Eqs. (34)-(36). Figure 9 shows the variation of the resonator sensitivity (Design1) with respect to the drive $D C$ voltage for two axial forces $\tilde{N}_{0}=10^{-6} \mathrm{~N}$ and $\tilde{N}_{0}=10^{-3} \mathrm{~N}$. The difference between both curves is negligible which ensures a large dynamic range inside which the scale factor is linear and is similar to the first order Taylor series expansion of Eq. (37). However, remarkably, the scale factor increases with respect to the drive voltage from $4.82 N^{-1}$ for $V_{d c}=1 V$ up to $5.1 N^{-1}$ for $V_{d c}=15 V\left(W_{M a x} \approx 0.95\right)$ which is due to the variation of the nonlinear stiffness with respect to applied axial force $\tilde{N}_{0}$ at large amplitudes. Indeed, the resonator sensitivity varies with respect to the level of nonlinearities as opposed to the linear case for which the scale factor of the resonant sensor is constant.

As expected and due to the scaling effects, the sensitivity of the second resonator is much higher than the first one which is shown in Fig. 10. Interestingly, the same investigations as before demonstrate the decrease of the scale factor with respect to the $D C$ voltage from $750 N^{-1}$ for $V_{d c}=1 \mathrm{~V}$ down to $630 \mathrm{~N}^{-1}$ for $V_{d c}=8 \mathrm{~V}$. This can be explained by the onset of a typical mixed hardening-softening behavior $[4,5,37]$ when the resonator is actuated using a $D C$ voltage beyond $5 \mathrm{~V}$. 


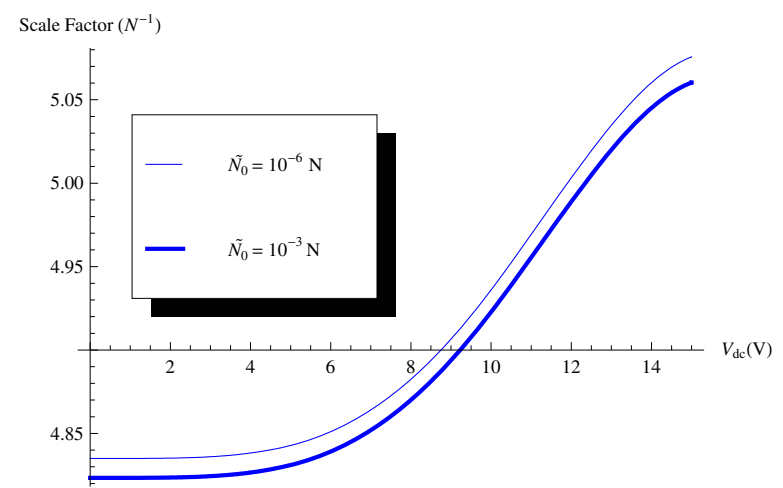

Figure 9: Variation of the resonator scale factor with respect to the drive $D C$ voltage for design $1\left(V_{a c}=0.1 V_{d c}\right)$

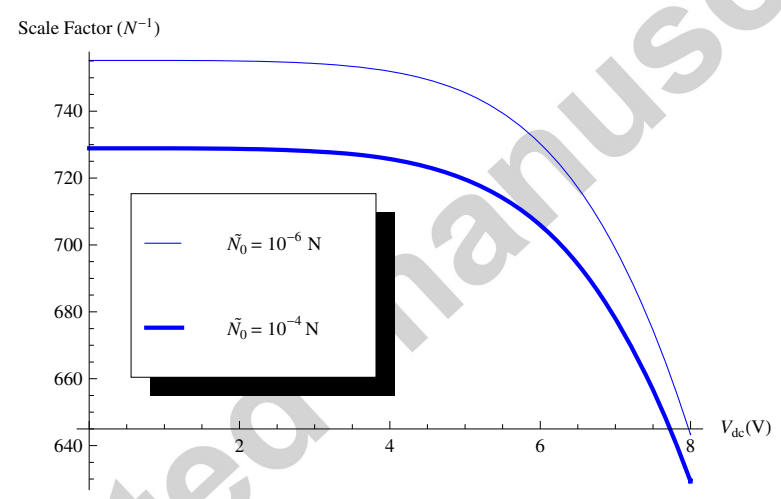

Figure 10: Variation of the resonator scale factor with respect to the drive $D C$ voltage for design $2\left(V_{a c}=0.1 V_{d c}\right)$

As a conclusion, for smaller ratio $g / h$ the sensitivity of the resonator to axial force is improved but can be drastically reduced at large displacements i.e. high actuation voltages.

\section{Conclusions}

In this paper, the nonlinear dynamics of MEMS and NEMS clamped-clamped beam resonators electrostatically actuated was modeled including the main sources of nonlinearities as well as the fringing field effects. The modal Galerkin decomposition method was used in order to transform the nonlinear Euler-Bernoulli PDE that governs the resonator motion into a system of a coupled nonlinear ODE. 
First, a multimodal numerical approach was developed by using the harmonic balance method (HBM) coupled with the so-called asymptotic numerical method (ANM), and validated with respect to a reference solution obtained by shooting.

Then, it was shown that the error made using only one mode is negligible compared to fabrication errors. A reduced order model neglecting the higher modes was therefore developed. To this end, the mode properties were investigated in order to simplify the matrices of the considered nonlinear system and to provide simplified analytical expressions for the Galerkin coefficients. Then, a perturbation method, namely the averaging method, was used to obtain closed-form expressions for the resonator dynamics which proved to be in very good agreement with the numerical results obtained with the HBM+ANM.

Moreover, parametric investigations can be easily performed. Analysis concerning the variation of the resonator sensitivity to axial force (in the case of a resonant accelerometer) demonstrates that when working in the nonlinear regime the scale factor is improved when the resonator is scaled down to the NEMS level, but it can be significantly reduced at high actuation voltages due to the soon onset of the mixed behavior. Remarkably, the variation of the scale factor with respect to the nonlinearity level was demonstrated. Consequently and in addition to the noise mixing issue [38], this is another motivation for the nonlinearity cancellation strategy $[4,37]$ in resonant MEMS and NEMS sensors.

More generally, this reduced-order analytical model is a fast but accurate tool for the prediction of the nonlinear behavior of MEMS and NEMS resonators. It can be used to derive simple design rules in order to enhance the performances of resonant sensors, which is of high interest for MEMS designers. On the other hand, although it is computationally more demanding, the numerical model is a powerful tool which can be used even when the dynamic response of the resonator is more complex.

\section{Appendix}

$\begin{array}{ll}\forall k \in \mathbb{N} & \int_{0}^{1}\left[\Phi_{k}^{\prime}\right]^{2} d x \simeq \frac{\pi(2 k+1)[(2 k+1) \pi-4]}{4} \\ \forall i \in \mathbb{N}^{*} & \int_{0}^{1} \Phi_{2 i-1} d x \simeq \frac{8}{\pi[1-4 i]}\end{array}$ 
$\forall i \in \mathbb{N}^{*} \quad \int_{0}^{1} \Phi_{2 i-1}^{3} d x \simeq \frac{64}{5 \pi(1-4 i)}$

$\forall i \in \mathbb{N}^{*} \quad \int_{0}^{1} \Phi_{2 i-1}^{\prime \prime} \Phi_{2 i-1}^{2} d x \simeq \frac{32 \pi(4 i-1)}{15}$

$\forall k \in \mathbb{N}^{*} \quad \int_{0}^{1} \Phi_{k}^{4} d x \simeq 3\left\{\frac{1}{\pi[2 k+1]}+\frac{1}{2}\right\}$

$\forall k \in \mathbb{N}^{*} \quad \int_{0}^{1} \Phi_{k}^{\prime \prime} \Phi_{k}^{3} d x \simeq\left[\frac{\pi}{2}+k \pi\right]\left\{0.9-1.5\left[\frac{\pi}{2}+k \pi\right]\right\}$

$\forall(i, k) \in \mathbb{N}^{*} \times \mathbb{N}^{*}$ and $\forall i \neq k$

$$
\begin{aligned}
& \int_{0}^{1} \Phi_{i}^{\prime} \Phi_{k}^{\prime} d x=-\int_{0}^{1} \Phi_{i} \Phi_{k}^{\prime \prime} d x \\
& \simeq \frac{4 \pi(i-k)\left[\left(\frac{1}{2}+i\right)+\left(\frac{1}{2}+k\right)\right]^{2}\left\{1+[-1]^{i+k}\right\}}{k(k+1)\left\{\frac{1}{2}+k(k+1)\right\}-i(i+1)\left\{\frac{1}{2}+i(i+1)\right\}}
\end{aligned}
$$

$\forall(i, k) \in \mathbb{N}^{*} \times \mathbb{N}^{*}$

$$
\begin{aligned}
& \int_{0}^{1} \Phi_{i} \Phi_{k}^{2} d x \simeq \frac{16\left\{1-[-1]^{i}\right\}}{\left(\frac{\pi}{2}+i \pi\right)\left(\frac{\pi}{2}+k \pi\right)^{-4}} \\
& \times \frac{\left\{8\left(\frac{\pi}{2}+k \pi\right)^{4}-3\left(\frac{\pi}{2}+i \pi\right)^{4}+10\left(\frac{\pi}{2}+i \pi\right)^{3}\left(\frac{\pi}{2}+k \pi\right)\right\}}{\left\{\left(\frac{\pi}{2}+i \pi\right)^{8}-12\left(\frac{\pi}{2}+i \pi\right)^{4}\left(\frac{\pi}{2}+k \pi\right)^{4}-64\left(\frac{\pi}{2}+k \pi\right)^{8}\right\}}
\end{aligned}
$$

$\forall n \in \mathbb{N}^{*} \quad \int_{0}^{1} \Phi_{2 n-1}^{3} d x \simeq \frac{64}{5 \pi(1-4 n)}$

$\forall(i, k) \in \mathbb{N}^{*} \times \mathbb{N}^{*}$

$$
\int_{0}^{1} \Phi_{i} \Phi_{k} \Phi_{k}^{\prime \prime} d x \simeq \frac{32\left([-1]^{i}-1\right)\left(\frac{\pi}{2}+k \pi\right)^{6}}{\left(\frac{\pi}{2}+i \pi\right)\left\{\left(\frac{\pi}{2}+i \pi\right)^{4}-16\left(\frac{\pi}{2}+k \pi\right)^{4}\right\}}
$$

$\forall n \in \mathbb{N}^{*} \quad \int_{0}^{1} \Phi_{2 n-1}^{\prime \prime} \Phi_{2 n-1}^{2} d x \simeq \frac{32 \pi(4 n-1)}{15}$

$\forall(i, k) \in \mathbb{N}^{*} \times \mathbb{N}^{*}$ and $\forall i \neq k$ 


$$
\left.\begin{array}{c}
\int_{0}^{1} \Phi_{i} \Phi_{k}^{3} d x \simeq \frac{1}{\pi} \times \\
\frac{3\left(1+[-1]^{i+k}\right)}{1+i+k}+\frac{1+[-1]^{i+3 k}}{2+i+3 k}+\frac{6\left(1+[-1]^{i+k}\right)(i-k)}{1+2 i+2 i^{2}+2 k+2 k^{2}} \\
-\frac{3\left(1+[-1]^{i+k}\right)(1+2 k)}{2+2 i+i^{2}+6 k+2 i k+5 k^{2}} \\
+\frac{4-2 i+10 k+3(1-i+3 k)[-1]^{i+k}+(1+i+k)[-1]^{i+3 k}}{2(-1+i-3 k)(1+i+k)} \\
-\frac{6\left(1+[-1]^{i+3 k}\right)(1+i+k)}{5+6 i+2 i^{2}+14 k+8 i k+10 k^{2}} \\
-\frac{3\left(1+[-1]^{i+3 k}\right)\left(-1-i+i^{3}-5 k-4 i k-3 i^{2} k-8 k^{2}-i k^{2}-5 k^{3}\right)}{\left(1+i^{2}+4 k-2 i k+5 k^{2}\right)\left(2+2 i+i^{2}+6 k+2 i k+5 k^{2}\right)} \\
-\frac{2\left(1+[-1]^{i+3 k}\right)(1+2 k)\left(-1+4 i+2 i^{2}-8 k+4 i k-10 k^{2}\right)}{\left(1-2 i+2 i^{2}+6 k-8 i k+10 k^{2}\right)\left(5+6 i+2 i^{2}+14 k+8 i k+10 k^{2}\right)} \\
+\frac{\left.2(1+1]^{i+3 k}\right)(-1+i-3 k)}{5+2 i+2 i^{2}+18 k+18 k^{2}}
\end{array}\right)
$$

$\forall(i, k) \in \mathbb{N}^{*} \times \mathbb{N}^{*}$ and $\forall i \neq k$ 


$$
\begin{aligned}
& \int_{0}^{1} \Phi_{i} \Phi_{k}^{2} \Phi_{k}^{\prime \prime} d x \simeq \frac{\pi(1+2 k)^{2}}{4} \times \\
& \begin{array}{c}
\frac{1+[-1]^{i+3 k}}{2+i+3 k}-\frac{2\left(1+[-1]^{i+k}\right)(i-k)}{1+2 i+2 i^{2}+2 k+2 k^{2}}+\frac{2\left(1+[-1]^{i+3 k}\right)(-1+i-3 k)}{5+2 i+2 i^{2}+18 k+18 k^{2}} \\
-\frac{(1+i+k)[-1]^{i+3 k}-3(-1+i-3 k)[-1]^{i+k}-2(-2+i-5 k)}{2(-1+i-3 k)(1+i+k)}-\frac{1+[-1]^{i+k}}{1+i+k} \\
+\frac{\left(1+[-1]^{i+k}\right)(1+2 k)}{2+2 i+i^{2}+6 k+2 i k+5 k^{2}}-\frac{2\left(1+[-1]^{i+3 k}\right)(1+i+k)}{5+6 i+2 i^{2}+14 k+8 i k+10 k^{2}} \\
+\frac{2\left(1+[-1]^{i+3 k}\right)(1+2 k)\left(-1+4 i+2 i^{2}-8 k+4 i k-10 k^{2}\right)}{\left(1-2 i+2 i^{2}+6 k-8 i k+10 k^{2}\right)\left(5+6 i+2 i^{2}+14 k+8 i k+10 k^{2}\right)} \\
+\frac{2\left(1+[-1]^{i+k}\right)\left(-1+5 i+2 i^{3}-11 k+20 i k-6 i^{2} k-32 k^{2}+26 i k^{2}-30 k^{3}\right)}{\left(1+2 i+2 i^{2}+2 k+2 k^{2}\right)\left(5+2 i+2 i^{2}+18 k+18 k^{2}\right)} \\
-\frac{\left(1+[-1]^{i+3 k}\right)\left(-1-i+i^{3}-5 k-4 i k-3 i^{2} k-8 k^{2}-i k^{2}-5 k^{3}\right)}{\left(1+i^{2}+4 k-2 i k+5 k^{2}\right)\left(2+2 i+i^{2}+6 k+2 i k+5 k^{2}\right)}
\end{array}
\end{aligned}
$$

\section{References}

[1] Y. Yang, C. Callegari, X. Feng, K. Ekinci, M. Roukes, Zeptogram-scale nanomechanical mass sensing, Nano Letters 6 (4) (2006) 583-586.

[2] M. Li, H. Tang, M. Roukes, Ultra-sensitive nems-based cantilevers for sensing, scanned probe and very highfrequency applications, Nature Nanotechnology 2 (2007) 114-120.

[3] K. Jensen, K. Kim, A. Zettl, An atomic-resolution nanomechanical mass sensor, Nature Nanotechnology 3 (2008) 533-537.

[4] N. Kacem, S. Hentz, D. Pinto, B. Reig, V. Nguyen, Nonlinear dynamics of nanomechanical beam resonators: improving the performance of nems-based sensors, Nanotechnology 20 (27) (2009) 275501.

[5] N. Kacem, J. Arcamone, F. Perez-Murano, S. Hentz, Dynamic range enhancement of nonlinear nanomechanical resonant cantilevers for high sensitive nems gas/mass sensors applications, Journal of Micromechanics and Microengineering 20 (2010) 045023 (9pp).

[6] W.-M. Zhang, G. Meng, D. Chen, Stability, nonlinearity and reliability of electrostatically actuated mems devices, Sensors 7 (5) (2007) 760-796.

[7] R. Batra, M. Porfiri, D. Spinello, Review of modeling electrostatically actuated microelectromechanical systems, Smart Materials and Structures 16 (6) (2007) R23-R31. 
[8] R. Lifshitz, M. Cross, Reviews of Nonlinear Dynamics and Complexity: Volume 1, Wiley-VCH, (2008), Ch. Nonlinear Dynamics of Nanomechanical and Micromechanical Resonators, pp. 1-52.

[9] S. Pamidighantam, R. Puers, K. Baert, H. Tilmans, Pull-in voltage analysis of electrostatically actuated beam structures with fixed-fixed and fixed-free end conditions, Journal of Micromechanics and Microengineering 12 (4) (2002) 458.

[10] L. Shao, M. Palaniapan, W. Tan, The nonlinearity cancellation phenomenon in micromechanical resonators, Journal of Micromechanics and Microengineering 18 (6) (2008) 065014 (9pp).

[11] H. Tilmans, R. Legtenberg, Electrostatically driven vacuum-encapsulated polysilicon resonators: Part ii. theory and performance, Sensors and Actuators A: Physical 45 (1) (1994) 67-84.

[12] E. Abdel-Rahman, M. Younis, A. Nayfeh, Characterization of the mechanical behavior of an electrically actuated microbeam, Journal of Micromechanics and Microengineering 12 (6) (2002) 759-766.

[13] M. Younis, E. Abdel-Rahman, A. Nayfeh, A reduced-order model for electrically actuated microbeam-based mems, Journal of Microelectromechanical Systems 12 (5) (2003) 672-680.

[14] M. Younis, A. Nayfeh, A study of the nonlinear response of a resonant microbeam to an electric actuation, Nonlinear Dynamics 31 (1) (2003) 91-117.

[15] W. Xie, H. Lee, S. Lim, Nonlinear dynamic analysis of mems switches by nonlinear modal analysis, Nonlinear Dynamics 31 (2003) 243-256.

[16] J.-H. Kuang, C.-J. Chen, Dynamic characteristics of shaped micro-actuators solved using the differential quadrature method, Journal of Micromechanics and Microengineering 14 (4) (2004) 647-655.

[17] F. Najar, S. Choura, S. El-Borgi, E. Abdel-Rahman, A. Nayfeh, Modeling and design of variable-geometry electrostatic microactuators, Journal of Micromechanics and Microengineering 15 (3) (2005) 419-429.

[18] A. Nayfeh, M. Younis, E. Abdel-Rahman, Dynamic pull-in phenomenon in mems resonators, Nonlinear Dynamics 48 (2007) 153-163.

[19] S. Krylov, Lyapunov exponents as a criterion for the dynamic pull-in instability of electrostatically actuated microstructures, International Journal of Non-Linear Mechanics 42 (4) (2007) 626-642.

[20] R. Batra, M. Porfiri, D. Spinello, Vibrations of narrow microbeams predeformed by an electric field, Journal of Sound and Vibration 309 (2008) 600-612.

[21] M. Moghimi Zand, M. Ahmadian, B. Rashidian, Semi-analytic solutions to nonlinear vibrations of microbeams under suddenly applied voltages, Journal of Sound and Vibration 325 (2009) 382-396.

[22] H. Nishiyama, M. Nakamura, Capacitance of a strip capacitor, IEEE Transactions on Components, Hybrids, and Manufacturing Technology 13 (2) (1990) 417-423.

[23] P. C.-P. Chao, C. Chiu, T.-H. Liu, Dc dynamic pull-in predictions for a generalized clamped-clamped microbeam based on a continuous model and bifurcation analysis, Journal of Micromechanics and Microengineering 18 (11) (2008) 115008 (14pp). 
[24] V. Rochus, D. J. Rixen, J. C. Golinval, Electrostatic coupling of mems structures: transient simulations and dynamic pull-in, Nonlinear Analysis 63 (2005) e1619-e1633.

[25] G. Michon, L. Manin, R. Rémond, R. Parker, R. Dufour, Parametric instability of an axially moving belt subjected to multi-frequency excitations: experiments and analytical validation, ASME Journal of Applied Mechanics 75 (2008) 041004 (8pp).

[26] S. Lau, Y. Cheung, Amplitude incremental variational principle for nonlinear vibration of elastic systems, ASME Journal of Applied Mechanics 28 (1981) 959-964.

[27] S. Nacivet, C. Pierre, F. Thouverez, L. Jezequel, A dynamic lagrangian frequency-time method for the vibration of dry-friction-damped systems, Journal of Sound and Vibration 265 (2003) 201-219.

[28] B. Cochelin, C. Vergez, A high order purely frequency-based harmonic balance formulation for continuation of periodic solutions, Journal of Sound and Vibration 324 (2009) 243-262.

[29] L. Azrar, B. Cochelin, N. Damil, M. Potier-Ferry, An asymptotic-numerical method to compute the postbuckling behaviour of elastic plates and shells, International Journal for Numerical Methods in Engineering 36 (1993) 1251-1277.

[30] B. Cochelin, A path-following technique via an asymptotic-numerical method, Computers and Structures 53 (1994) 1181-1192.

[31] S. Baguet, B. Cochelin, On the behaviour of the anm in the presence of bifurcations, Communications in Numerical Methods in Engineering 19 (2003) 459-471.

[32] B. Cochelin, N. Damil, M. Potier-Ferry, Méthode asymptotique Numérique, Collection méthodes numériques, Hermes Sciences Lavoisier (2007) (in French).

[33] A. Nayfeh, B. Balachandran, Applied Nonlinear Dynamics: Analytical, Computational, and Experimental Methods, Nonlinear Science, Wiley, 1995.

[34] R. Dufour, A. Berlioz, Parametric instability of a beam due to axial excitations and to boundary conditions, ASME Journal of Vibration and Acoustics 120 (1998) 461-467.

[35] K. Brueckner, V. Cimalla, F. Niebelschütz, R. Stephan, K. Tonisch, O. Ambacher and M. A. Hein, Strainand pressure-dependent RF response of microelectromechanical resonators for sensing applications, Journal of Micromechanics and Microengineering 17 (10) (2007) 2016.

[36] V. Sazonova, A tunable carbon nanotube resonator, Ph.D. Dissertation, Cornell University, Ithaca, New York, 2006.

[37] N. Kacem, S. Hentz, Bifurcation topology tuning of a mixed behavior in nonlinear micromechanical resonators, Applied Physics Letters 95 (18) (2009) 183104.

[38] V. Kaajakari, J. Koskinen, T. Mattila, Phase noise in capacitively coupled micromechanical oscillators, Ultrasonics, Ferroelectrics and Frequency Control, IEEE Transactions on 52 (12) (2005) 2322-2331. 